\title{
Cadaveric Study of Angiosomes of Anterior Tibial and Dorsalis Pedis Artery
}

\author{
$\begin{array}{llll}\text { V. Paranjape } & \text { J. Kulkarni } & \text { P.V. Swamy } & \text { S. Shevade }\end{array}$ \\ ${ }^{1}$ Department of Anatomy, Dr. D. Y. Patil Medical College Hospital and \\ Research Centre, Dr. D. Y. Patil Univrsity, Sant Tukaram Nagar, \\ Pimpri, Pune, Maharashtra, India \\ 2 Department of Anatomy, Sikkim Manipal University, 5th Mile, \\ Tadong, Gangtok, Sikkim, India \\ ${ }^{3}$ Dr. D. Y. Patil Medical College Hospital and Research Centre, \\ Dr. D. Y. Patil University, Sant Tukaram Nagar, Pimpri, Pune, \\ Maharashtra, India
}

J Morphol Sci 2018;35:17-24.

\begin{abstract}
Keywords

- dorsalis pedis artery

- angiosome

- vascular surgery

- lateral tarsal artery

- myocutaneous graft

Introduction Angiosome formed by Anterior tibial artery and dorsalis pedis artery supply the ankle and the dorsum of foot. Both the vessels shows variation in termination and branching pattern.

Materials and Methods 50 free formalin preserved limbs were dissected for the study.

Results and Conclusion Anterior tibial artery showed variation in termination in 16\%, most common of them was formation of loop with perforating branch of peroneal artery in $10 \%$, bifurcation in to medial and lateral tarsal artery in $4 \%$ and trifutcation in $2 \%$. Branching pattern of dorsalis pedis artey was variable in $18 \%$, medial and lateral tarsal arteries were not branches of dorsalis pedis artery in $14 \%$ and $16 \%$ respectively. Arcuate artery was absent in $10 \%$, when present it was branch of dorsalis pedis artery in $96 \%$, perforating branch of peroneal artery in $2 \%$ and loop of medial and lateral tarsal artery in $2 \%$. First dorsal metatarsal artery was not branch of dorsalis pedis artery in $4 \%$. Lateral tarsal artery was constantly found in all the dissected limbs. Thorough knowledge of variation about branching pattern of arterial tree is clinically important for accuracy in interpretation of angiographs, Doppler study of lower limb vessels and optimum use of vascular pedicle for myocutaneous flaps. Importance of palpation of peripheral pulse in lateral tarsal artery is discussed in the study.
\end{abstract}

Address for correspondence Dr. V. Paranjape, Department of Anatomy, Dr. D. Y. Patil Medical College Hospital and Research Centre, Dr. D. Y. Patil University, Sant Tukaram Nagar, Pimpri, Pune, Maharashtra, 411018, India (e-mail: vmp1997@gmail.com).

\section{Introduction}

Anterior tibial artery (ATA), Posterior tibial (PTA) artery and Dorsalis pedis (DPA) artery are routinely used to access peripheral pulsation of lower extremity. Anterior Tibial (ATA) artery is a terminal branch of Popliteal artery. It approaches ankle joint midway between the two malleoli deep to superior extensor retinaculum, then lies between the tendons of Extensor hallucis longus and Tibialis anterior accompanied by Deep peroneal nerve on its lateral aspect. It continues distal to ankle joint as Dorsalis pedis artery (DPA).

Medial and Lateral malleolar ananstomotic network is formed by branches of Posterior tibial artery (PTA), Peroneal artery (PA), Anterior tibial artery (ATA), and Dorsalis pedis artery (DPA).

Anterior Medial malleolar artery (AMMA) and Anterior Lateral Malleolar Artery (ALMA) are the branches of ATA. received

May 28, 2015

accepted

February 10, 2018
DOI https://doi.org/

10.1055/s-0038-1660483. ISSN 2177-0298.
Copyright $(2018$ by Thieme Revinter

Publicações Ltda, Rio de Janeiro, Brazil
License terms

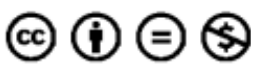


Peroneal artery is branch of Posterior tibial artery. Perforating branch of Peroneal artery (PBPA) anastomoses with Anterior lateral malleolar artery (ALMA) and Lateral tarsal artery (LTA). Dorsalis pedis (DPA) artery passes deep to inferior extensor retinaculum and gives Medial Tarsal Arteries (MTA) and Lateral Tarsal Artery (LTA). Dorsalis Pedis gives Arcuate artery (AA) and First Dorsal Metatarsal Artery (FDMA) and Dorsalis pedis itself enters the sole to complete Plantar arch by anastomosing with the branches of Posterior Tibial artery. Branches of DPA artery contribute to the formation of malleolar ananstomosis and also contribute to formation of plantar arch. This makes DP artery an important contributor to limb salvage surgery.

Second (SDMA), third and fourth dorsal metatarsal arteries are the branches of Arcuate artery (AA).

Here we present the branching pattern of Angiosome on dorsum of foot formed by Anterior tibial artery and Dorsalis pedis artery on dorsum of foot.

\section{Materials and Methods}

50 Free Formalin preserved limbs of unknown sex and age were taken from Department of Anatomy Dr DY Patil Mediacl College Hospital and Research center Pimpri, Pune; and dissected. Vertical incision was taken on anterior aspect of leg five centimeters above the anterior aspect of ankle joint midway between the lateral and medial malleoli. The incision was extended on dorsum of foot till base of the second toe. Distal limit of the incision was extended on both the sides till the lateral and medial border of the foot. Skin flaps were reflected. Anterior Tibial artery was identified between tendons of Tibialis anterior and Extensor hallucis longus and studied with respect to its termination along with the branching pattern of dorsalis pedis artery pattern on the dorsum of foot. Arterial tree distal to termination of Anterior Tibial artery was carefully dissected and observations were tabulated. The limbs showing gross anomalies were excluded from the study. The research was conducted according to the principles of the Declaration of Helsinki.

\section{Results}

The results are tabulated in $\boldsymbol{- T a b l e} \mathbf{1}$.

Anterior tibial artery (ATA) continued as dorsalis pedis artery in $42 / 50$ limbs (84\%).

Anterior tibial artery showed variation at its termination in $8 / 50(16 \%)$ limbs as follows:

Anterior tibial artery bifurcated in medial tarsal (MT) and lateral tarsal (LT) in 2/8 limbs.

Anterior tibial artery trifurcated in anterior lateral malleolar (ALMA), LT, dorsalis pedis (DPA) in $1 / 8$ limbs.

Anterior Tibial artery anastomosed with the perforating branch of peroneal artery (PBPA) in $5 / 8$ limbs.

AMMA (Anterior medial malleolar artery) was branch of Anterior tibial artery in $47 / 50$ limbs (94\%).

AMMA was not a branch of ATA in 3/50 (6\%) limbs in such cases; AMMA was branch of DPA in $1 / 3$ limbs.
AMMA was branch of loop formed by ATA and PBPA in 2/3 limbs.

Anterior lateral malleolar artey (ALMA) was branch of Anterior tibial artery in $45 / 50$ (90\%) limbs.

When, ALMA was not a branch of ATA in 5/50 (10\%) limbs, in such cases;

ALMA was branch of PP in 5/50 (10\%) limbs.

MTA (Medial tarsal artery) was branch of DP (dorsalis pedis) $43 / 50$ (86\%) limbs.

When, MTA was not a branch of DPA in 7/50 (14\%) limbs, in such cases;

MTA was branch of ATA in 2/7 limbs.

MTA was branch of loop formed by ATA and PBPA in 5/7 limbs.

LTA (lateral tarsal artery) was a branch of DPA in $42 / 50$ (84\%) limbs.

When, LTA was not a branch of DPA in 8/50 (16\%) limbs, in such cases;

LTA was branch of ATA in $3 / 8$ limbs.

LTA was branch of Loop formed by ATA and PBPA in 2/8 limbs.

LTA was branch of PBPA in $3 / 8$ limbs.

DPA (dorsalis pedis artery) was continuation of ATA in 43/ 50 (86\%) limbs.

When, DPA was not continuation of ATA in $7 / 50$ (14\%) limbs, in such cases;

DPA was branch of MTA in 2/7 limbs. DPA was branch of LTA in $1 / 7$ limbs.

DPA was branch of loop formed by ATA and (PBPA) in 3/7 limbs.

DPA was a branch of PP in 1/7 limbs.

AA (arcuate artery) was present in $45 / 50$ (90\%) limbs. AA was absent in 5/50 limbs (10\%).

AA was branch of DPA in 43/45 (86\%) limbs.

AA was branch of loop formed by MT and LT in $1 / 45$ limbs. AA was branch of PBPA in 1/45 limb.

FDMTA (First dorsal metatarsal artery) was branch of DPA in $48 / 50$ (96\%) limbs.

FDMA was not a branch of DPA in $2 / 50$ (4\%) limbs. FDMA was a branch of loop formed by ATA and PBPA $1 / 2$ limbs.

FDMA was branch of LTA in $1 / 2$ limbs.

SDMA (second dorsal metatarsal artery) was branch of AA in $44 / 50(88 \%)$ limbs.

SDMA was not a branch of AA in 6/50 (12\%) limbs. SDMA was a branch of LTA in 2/7 limbs.

SDMA was a branch of DPA in 2/7 limbs.

SDMA was branch of loop formed by ATA and PBPA in 2/6 limbs.

Variation in termination of ATA was seen in $8 / 50$ limbs $16 \%$ of the limbs.

Variation in branching pattern of DPA was seen in $18 \%$ of the limbs.

Dorsalis pedis artery showed variation with respect to its origin and branching pattern as follows:

DPA was not continuation of ATA in $14 \%$.

DPA did not give MTA and LTA in 14\% and 16\% respectively.

AA was absent in $10 \%$. 
Table 1 Branching Pattern of Anterior Tibial Artery

\begin{tabular}{|c|c|c|c|c|c|c|c|c|c|c|}
\hline Sp no & side & $\begin{array}{l}\text { ATA } \\
\text { continues as }\end{array}$ & $\begin{array}{l}\text { AMMA P/A } \\
\text { branch of }\end{array}$ & $\begin{array}{l}\text { ALMA P/A } \\
\text { branch of }\end{array}$ & $\begin{array}{l}\text { MTA P/A } \\
\text { branch of }\end{array}$ & $\begin{array}{l}\text { LTA P/A } \\
\text { branch of }\end{array}$ & $\begin{array}{l}\text { DPA P/A } \\
\text { continuation of }\end{array}$ & $\begin{array}{l}\text { AA P/A } \\
\text { branch of }\end{array}$ & $\begin{array}{l}\text { FDMA } \\
\text { branch of }\end{array}$ & $\begin{array}{l}\text { SDMA } \\
\text { branch of }\end{array}$ \\
\hline 1 & $\mathrm{R}$ & bifurcates & ATA p & ATA p & ATA p & ATA p & MT p & A nil & LT & LT \\
\hline 2 & $\mathrm{R}$ & anast PBPA & ATA p & PBPA p & loop p & loop p & PP P & A nil & DPA & DPA \\
\hline 3 & $\mathrm{~L}$ & DPA & ATA p & ATA p & DPA p & DPA p & ATA p & A nil & DPA & DPA \\
\hline 4 & $\mathrm{R}$ & DPA & ATA p & ATA p & DPA p & DPA p & ATA p & P DPA & DPA & $\mathrm{AA}$ \\
\hline 5 & $\mathrm{R}$ & DPA & ATA p & ATA p & DPA p & DPA p & ATA p & P DPA & DPA & AA \\
\hline 6 & $\mathrm{R}$ & DPA & ATA p & ATA p & DPA p & DPA $p$ & ATA p & P DPA & DPA & $A A$ \\
\hline 7 & $\mathrm{~L}$ & DPA & ATA p & ATA p & DPA $p$ & DPA p & ATA p & P DPA & DPA & $\mathrm{AA}$ \\
\hline 8 & $\mathrm{R}$ & DPA & ATA p & ATA p & DPA p & DPA p & ATA p & P DPA & DPA & $A A$ \\
\hline 9 & $\mathrm{~L}$ & DPA & ATA p & ATA p & DPA p & DPA p & ATA p & P DPA & DPA & AA \\
\hline 10 & $\mathrm{R}$ & anast PBPA & loop p & PBPA p & loop p & PBPA p & loop p & P DPA & DPA & $A A$ \\
\hline 11 & $\mathrm{~L}$ & anast PBPA & loop p & PBPA p & loop p & PBPA p & loop p & P DPA & DPA & $\mathrm{AA}$ \\
\hline 12 & $\mathrm{R}$ & DPA & ATA p & ATA p & DPA p & DPA p & ATA p & P DPA & DPA & AA \\
\hline 13 & $\mathrm{~L}$ & DPA & ATA p & ATA p & DPA p & DPA p & ATA p & P DPA & DPA & AA \\
\hline 14 & $\mathrm{R}$ & DPA & ATA p & ATA p & DPA p & DPA p & ATA p & P DPA & DPA & AA \\
\hline 15 & $\mathrm{~L}$ & DPA & ATA p & ATA p & DPA p & DPA p & ATA p & P DPA & DPA & $A A$ \\
\hline 16 & $R$ & DPA & ATA p & ATA p & DPA $p$ & DPA p & ATA p & P DPA & DPA & $\mathrm{AA}$ \\
\hline 17 & $\mathrm{~L}$ & DPA & ATA p & ATA p & DPA p & DPA p & ATA p & P DPA & DPA & $\mathrm{AA}$ \\
\hline 18 & $\mathrm{R}$ & DPA & ATA p & ATA p & DPA p & DPA p & ATA p & P DPA & DPA & $A A$ \\
\hline 19 & $\mathrm{~L}$ & DPA & ATA p & ATA p & DPA p & DPA p & ATA p & P DPA & DPA & $A A$ \\
\hline 20 & $\mathrm{~L}$ & DPA & ATA p & ATA p & DPA p & DPA p & ATA p & P DPA & DPA & $\mathrm{AA}$ \\
\hline 21 & $\mathrm{~L}$ & DPA & ATA p & ATA p & DPA p & ATA p & ATA p & P DPA & DPA & AA \\
\hline 22 & $\mathrm{R}$ & trifurcates & DPA p & ATA p & DPA p & DPA p & ATA p & A nil & DPA & LTA \\
\hline 23 & $\mathrm{R}$ & DPA & ATA p & ATA p & DPA p & DPA p & ATA p & P DPA & DPA & $A A$ \\
\hline 24 & $\mathrm{~L}$ & DPA & ATA p & ATA P & DPA $p$ & DPA p & ATA p & P DPA & DPA & $\mathrm{AA}$ \\
\hline 25 & $\mathrm{R}$ & DPA & ATA p & ATA p & DPA p & DPA p & ATA p & P DPA & DPA & AA \\
\hline 26 & $\mathrm{~L}$ & DPA & ATA p & ATA p & DPA p & DPA p & ATA p & P DPA & DPA & AA \\
\hline 27 & $\mathrm{R}$ & DPA & ATA p & ATA P & DPA p & DPA p & ATA p & P DPA & DPA & $A A$ \\
\hline 28 & $\mathrm{R}$ & DPA & ATA p & ATA p & DPA p & DPA p & ATA p & P DPA & DPA & $A A$ \\
\hline 29 & $R$ & anast PBPA & ATA p & ATA p & loop p & loop p & LTA P & A nil & DPA & DPA \\
\hline 30 & $\mathrm{~L}$ & DPA & ATA p & ATA P & DPA p & DPA p & ATA p & P DPA & DPA & $A A$ \\
\hline 31 & $\mathrm{~L}$ & DPA & ATA p & ATA P & DPA p & DPA p & ATA p & P DPA & DPA & AA \\
\hline 32 & $\mathrm{R}$ & DPA & ATA p & ATA p & DPA p & DPA p & ATA p & P DPA & DPA & $A A$ \\
\hline 33 & $R$ & DPA & ATA p & ATA p & DPA p & DPA p & ATA p & P DPA & DPA & $\mathrm{AA}$ \\
\hline 34 & $\mathrm{~L}$ & DPA & ATA p & ATA p & DPA p & DPA p & ATA p & P DPA & DPA & AA \\
\hline 35 & $\mathrm{R}$ & bifurcates & ATA p & ATA p & ATA p & ATA p & MTA p & P loop MTA- LTA & loop & loop \\
\hline 36 & $\mathrm{~L}$ & DPA & ATA p & ATA P & DPA p & DPA p & ATA p & P DPA & DPA & AA \\
\hline 37 & $\mathrm{~L}$ & DPA & ATA p & ATA p & DPA p & DPA p & ATA p & P DPA & DPA & $A A$ \\
\hline 38 & $\mathrm{~L}$ & DPA & ATA p & ATA p & DPA p & DPA p & ATA p & P DPA & DPA & $\mathrm{AA}$ \\
\hline 39 & $\mathrm{R}$ & DPA & ATA p & ATA p & DPA p & DPA p & ATA p & P DPA & DPA & $A A$ \\
\hline 40 & $\mathrm{R}$ & DPA & ATA p & ATA p & DPA p & DPA p & ATA p & P DPA & DPA & AA \\
\hline 41 & $\mathrm{~L}$ & DPA & ATA p & ATA p & DPA p & DPA p & ATA p & P DPA & DPA & $A A$ \\
\hline 42 & $\mathrm{R}$ & DPA & ATA $p$ & ATA p & DPA p & DPA p & ATA p & P DPA & DPA & AA \\
\hline 43 & $\mathrm{R}$ & anast PBPA & $\begin{array}{l}\text { ATA - UP } \\
\text { branch P }\end{array}$ & $\begin{array}{l}\text { PBPA LO } \\
\text { branch P }\end{array}$ & $\begin{array}{l}\text { ATA UB } \\
\text { branch P }\end{array}$ & $\begin{array}{l}\text { PBPA LO } \\
\text { branch P }\end{array}$ & ATA P & P DPA & DPA & $A A$ \\
\hline 44 & $\mathrm{R}$ & DPA & ATA p & ATA p & DPA p & DPA p & ATA p & P DPA & DPA & AA \\
\hline 45 & $\mathrm{~L}$ & DPA & ATA p & ATA p & DPA p & DPA p & ATA p & P DPA & DPA & $A A$ \\
\hline 46 & $R$ & DPA & ATA p & ATA p & DPA p & DPA p & ATA p & P DPA & DPA & $\mathrm{AA}$ \\
\hline 47 & $\mathrm{~L}$ & DPA & ATA p & ATA p & DPA p & DPA p & ATA p & P DPA & DPA & AA \\
\hline
\end{tabular}


Table 1 (Continued)

\begin{tabular}{|l|l|l|l|l|l|l|l|l|l|l|}
\hline Sp no & side & $\begin{array}{l}\text { ATA } \\
\text { continues as }\end{array}$ & $\begin{array}{l}\text { AMMA P/A } \\
\text { branch of }\end{array}$ & $\begin{array}{l}\text { ALMA P/A } \\
\text { branch of }\end{array}$ & $\begin{array}{l}\text { MTA P/A } \\
\text { branch of }\end{array}$ & $\begin{array}{l}\text { LTA P/A } \\
\text { branch of }\end{array}$ & $\begin{array}{l}\text { DPA P/A } \\
\text { continuation of }\end{array}$ & $\begin{array}{l}\text { AA P/A } \\
\text { branch of } \\
\text { branch of }\end{array}$ & $\begin{array}{l}\text { SDMA } \\
\text { branch of }\end{array}$ \\
\hline 48 & L & DPA & ATA p & ATA p & DPA p & DPA p & ATA p & P DPA & AA \\
\hline 49 & R & DPA & ATA p & ATA p & DPA p & DPA p & ATA p & P DPA & DPA \\
\hline 50 & L & DPA & ATA p & ATA p & DPA p & DPA p & ATA p & P DPA \\
\hline
\end{tabular}

Abbreviations: A, absent; AA, arcuate artery; ALMA P/A, anterior lateral malleolar artery; AMMA P/A, anterior medial malleolar artery; anast PBPA, anastomosis with perforating branch of peroneal artery; ATA - UP Branch; P, anterior tarsal artery; Upper Branch, present; ATA, anterior tibial artery; DPA, dorsalis pedis artrery; FDMA, first dorsal metatarsal artery; LTA, lateral tarsal artery; MTA, medial tarsal artery; P, present; PBPA, LO branch, PPerforating Branch of Peroneal Artery, Lower Branch, Present; SDMA, second dorsal metatarsal artery; Sp no, specimen number.

When present AA it was not a branch of DPA in $4 \%$ limbs. DP did not give FDMA in $4 \%$ limbs.

Extra branch in form of AMMA was branch of DPA in 2\% limbs. Extra branch in form of SDMT was branch of DPA in $4 \%$ limbs.

Thus variations found in the above study can be grouped as: Type A:

Most common variation ( $5 / 8,62.5 \%$ of the variations seen) seen in our study was formation of a loop by perforating branch of peroneal artery and anterior tibial artery. In $4 / 5$ (80\%) limbs with this type of variation, anterior tibial artery was rudimentary and thin where as the perforating branch of peroneal artery was the prominent and larger and formed main source of supply to dorsum of foot. In $1 / 5$ (20\%) ATA and

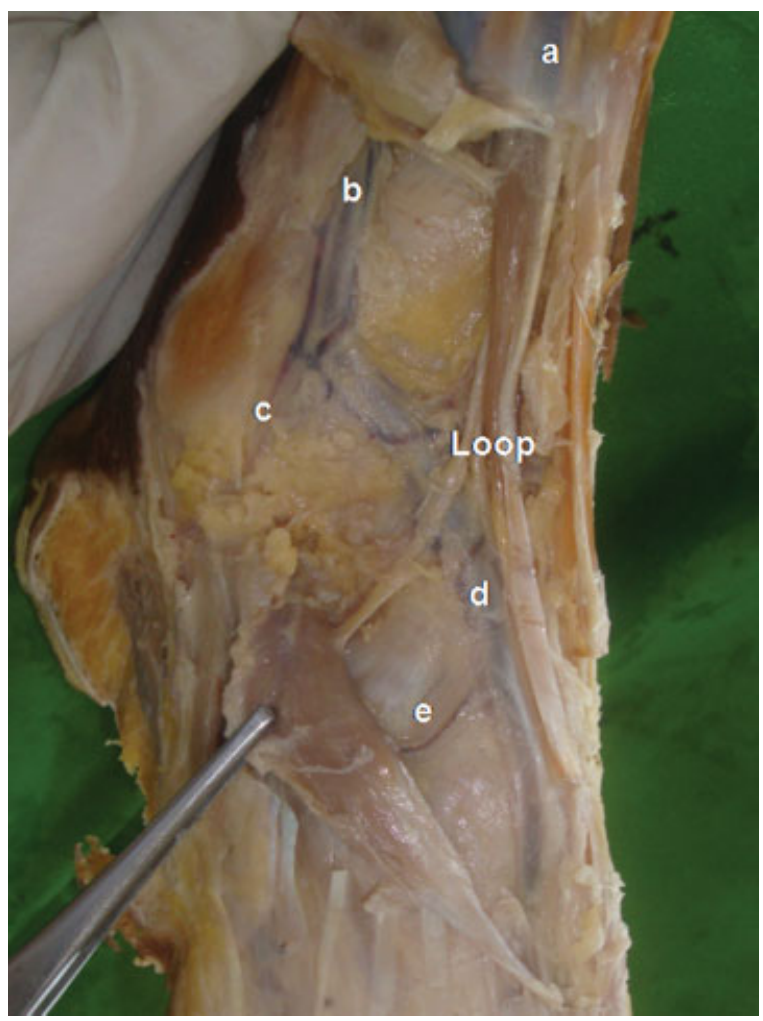

Fig. 1 (a) Anterior Tibial Artery (ATA); (b) Perforating Branch of Peroneal Artery (PBPA); (c) Lateral Tarsal Artery (LTA); (d) Dorsalis Pedis Artery (DPA); (e) Arcuate Artery (AA), Loop- Loop formed by ATA and PBPA. branches or PBPA itself were equally dominant and formed a loop. Branches from loop further supplied dorsum of foot.

Type B:

ATA did not continue as DP but terminated. (ATA bifurcated or trifurcated in our study). ATA bifurcated in MT and LT in two right limbs $(2 / 8,25 \%$ of the variations). Pattern of bifurcation was different in both the cases.

ATA trifurcated in one right $\operatorname{limb}(1 / 8,12.5 \%$ of the variations). Examples of these variations are as follows: Type A:

In all cases, Ananstamotic loop was formed in the vicinity of ankle joint. Loop once formed showed different type of branching pattern when traced further as seen in specimen no 10 - Fig. 1 and specimen 43 - Fig. 2.

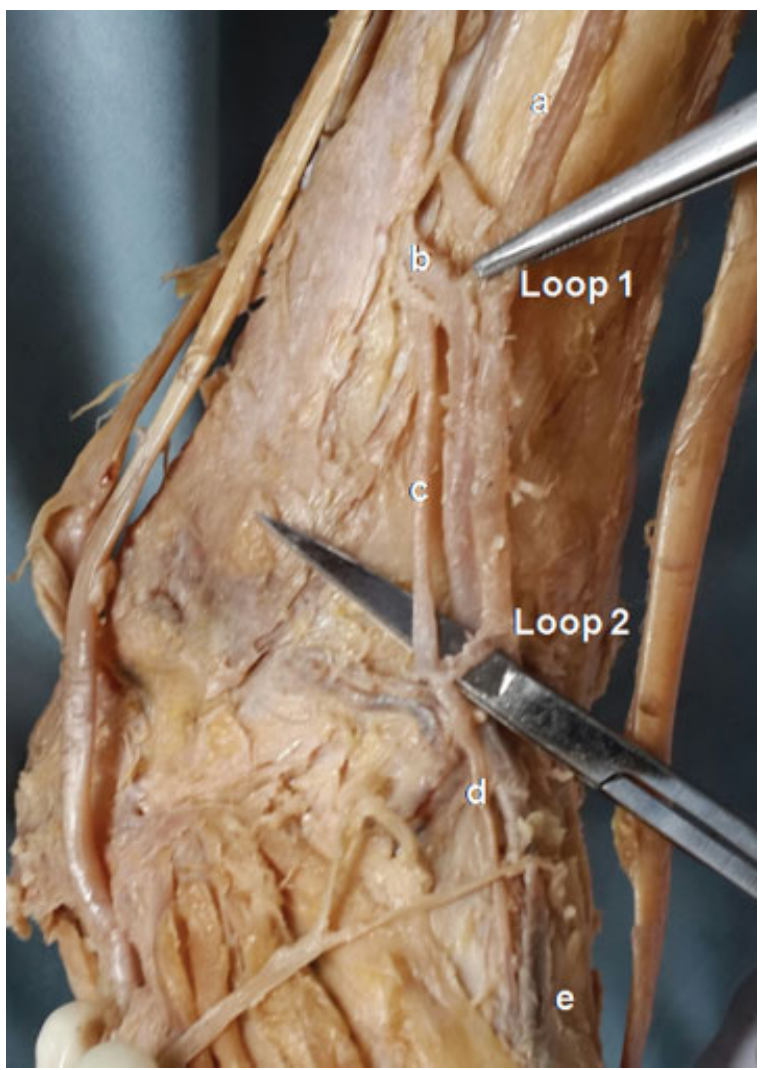

Fig. 2 (a) Anterior Tibial Artery (ATA); (b) Perforating Branch of Peroneal Artery, UB) upper branch (PBPA); (c) (LB) Lower branch of PBPA; (d) Dorsalis Pedis Artery (DPA); (e) First Dorsal Meta tarsal Artery (FDMA). Loop 1: Upper branch of PBPA and ATA; Loop 2: Lower branch of PBPA and ATA. 


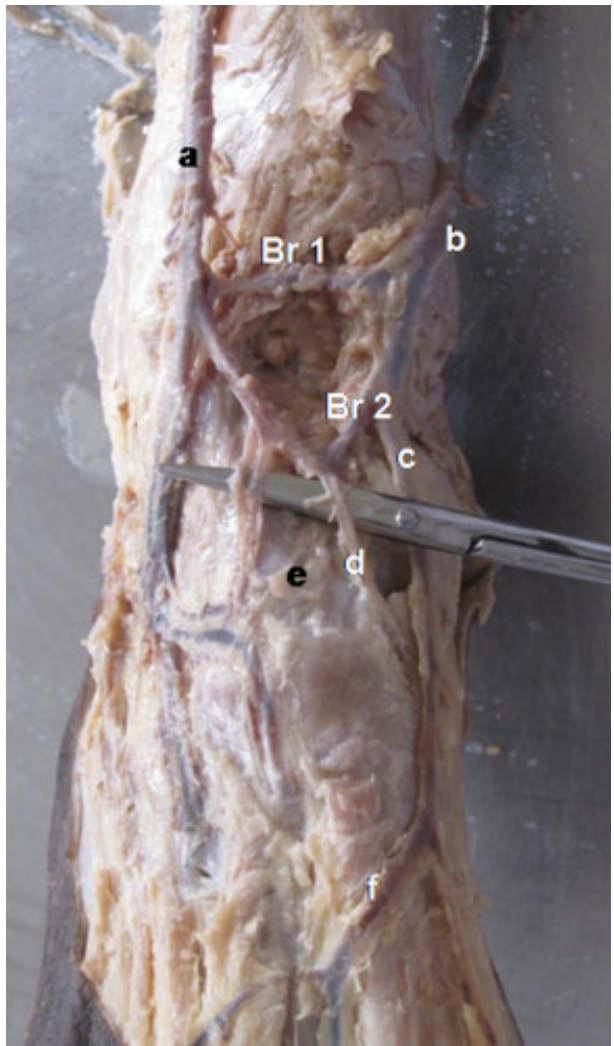

Fig. 3 (a) Perforating Branch Of Peroneal Artery (PBPA);(b) Anterior Tibial Artery (ATA); (c) Medial Tarsal Artery (MTA);(d) Dorsalis Pedis Arery (DPA); (e) Second Dorsal Metatarsal Artery (SDMA); (f) First Dorsal Metatarsal Artery; $\mathrm{Br}$ 1: First branch of ATA; $B r 2$ : Second branch of ATA.

-Fig. 1: specimen no 10; - Table 1: ATA was rudimentary and PBPA was dominant vessel. DP artery was continuation of PBPA and entered first intermetatarsal space. LTA was branch PBPA. MTA was branch of loop. Further branching pattern of DP was normal.

-Fig. 2: specimen no 43; - Table 1: ATA and PBPA, both vessels were dominant. Anastomosis between PBPA and ATA was seen at two levels. PBPA gave two branches (upper branch UB and lower branch LB) $6 \mathrm{~cm}$ above the ankle joint. Upper branch (UB) of PBPL anastomosed with a small branch from ATA at the same level. ATA continued distally toward ankle joint and gave another branch which anastomosed with lower branch (LB) of PBPL at the level of Ankle joint. LB of PBPA continued as DP artery. LTA was seen arising at the level of lower anastomosis. ATA deviated medially and continued as MTA. Further branching pattern of DPA was normal.

-Fig. 3: specimen no 35; - Table 1: In this case ATA did not continue as DPA. ATA bifurcated and also anastomosed with PBPA. PBPA and ATA were prominent. ATA bifurcated at the level of ankle joint. Both branches of ATA anastomosed with PBPA. Anastomosis was noted at two different levels. One branch anastomosed with PBPA at the same level as bifurcation. Second branch anastomosed with PBPA which further continued as DPA. DPA continued as FDMA. PBPA before ananstomosing with second branch of ATA gave

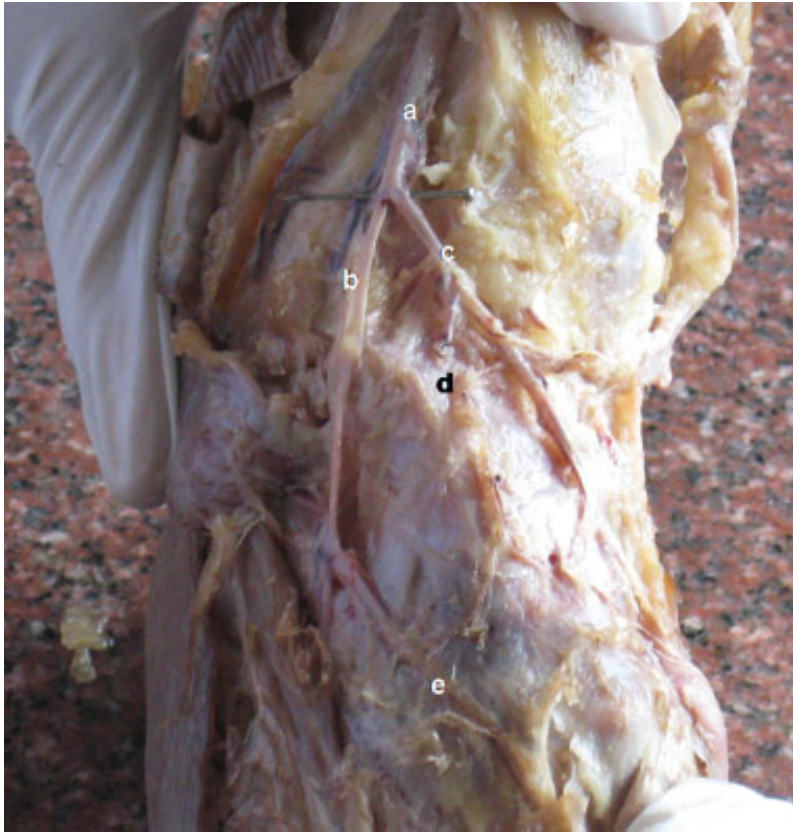

Fig. 4 (a) Anterior Tibial Artery (ATA); (b) Lateral Tarsal Artery (LTA); (c) Medial Tarsal Artery (MTA); (d) Dorsalis Pedis Artery (DPA); (e) First Dorsal Metatarsal Artery (FDMA).

another branch which gave SDMA and other metatarsal arteries. MTA and LTA were branches of ATA.

Type B:

ATA terminated instead of continuing as DPA.

-Fig. 4: specimen no 1; - Table 1: ATA terminated as it bifurcated in MTA and LTA. DPA was branch of MTA. MTA was further traversed along medial border of foot and entered sole. DPA was very thin and rudimentary. LTA was branch ATA and gave all dorsal metatarsal arteries. Arcuate artery was absent here.

-Fig. 5: specimen no 22; - Table 1: ATA trifurcated in LMA, LTA and DPA. MMA and MTA were branched of DPA. FDMA was branch of DPA. SDMA was branch of LTA. Arcuate artery was absent.

\section{Discussion}

Knowledge of vascular anatomy of foot is of outmost importance in clinical medicine. Absence or abnormality of peripheral pulsation suggests some kind vascular disease. Vascular disease can be in the form of thromboanginitis obliterans as in Burgers disease or microvascular angiopathy associated with diabetes or atherosclerotic changes. Variation in normal anatomy and branching pattern of ATA as evident from various studies is also one of the reasons for abnormal or absent peripheral pulsation. Successful treatment of pathology related to foot depends on prevention of tissue ischemia by establishment of sufficient vascular supply to distal fore foot. This is possible only with competent and viable ananstomosis of distal and proximal foot vessels via bypass graft of occluded segment or endovascular interventions like percutaneous transluminal angioplasty and stents. Not only for treatment of vascular diseases, ATA and DPA are also used as chief vessel 


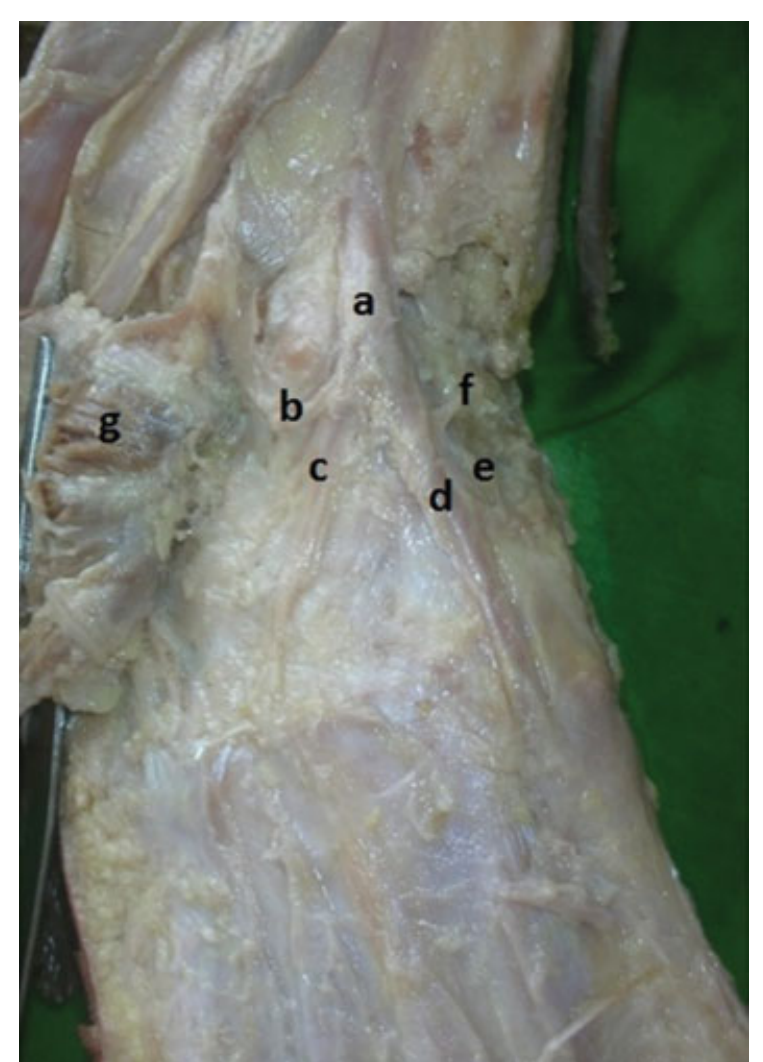

Fig. 5 (a) Anterior Tibial Artery (ATA); (b) Anterior Lateral Malleolar Artery (ALMA); (c) Lateral Tarsal Artery (LTA); (d) First Dorsal Metatarsal Artery; (e) Medial Tarsal Artery (MTA); (f) Anterior Medial Malleolar Artery (AMMA). for pedicle or free myocutaneous graft of extensor digitorum brevis muscle. Whichever is the modality of treatment, thorough knowledge vascular anatomy is essential. Blood vessels supplying foot and ankle are divided in to 6 angiosomes supplied by three branches of posterior tibial artery (supplying plantar aspect of foot), two branches of peronel artery (supplying foot and ankle from anterolateral aspect) and ATA and its continuation DPA (supplying ankle and dorsum of foot $)^{1}$ Anterior tibial artery (ATA) continued as Dorsalis pedis (DPA) in $74 \%,{ }^{2} 86 \%,{ }^{3} 92 \%,{ }^{4} 54.76 \%,{ }^{5} 24.1 \%{ }^{6}$ and $86 \%$ as seen in our study. In $16 \%$ of the limbs studied by us, DPA was not continuation of ATA ( - Table 2 ).

DPA was formed by equal contribution from ATA and PBPA in $4 \%$ (Swathi, 2010), ${ }^{2} 6 \%$ in our study, in such cases we found formation of a loop contributed by PBPA and ATA at the level of ankle joint, all distal branches were given from the loop including DPA, MTA and LTA; hence branching pattern of entire arterial tree on dorsum of foot was different. This pattern is similar to Type E pattern described by Vasudha and Ramesh $(2012)^{6}$ in $12.1 \%$ cases. In such cases Anterior tibial and Peroneal arteries have equal contribution to blood supply on dorsum of foot. Other studies do not mention about this type of branching pattern (-Table 2).

DPA was branch of PBPA as follows: In $16 \%,{ }^{2} 8 \%{ }^{4} 6.7 \%^{3}$ and $2 \%$ in our study (We have excluded those limbs where DPA was branch of loop formed by ATA and PBPA 6\%). Isolated cases of PBPA continuing as DPA has been reported by Tuncel et al., ${ }^{11} \mathrm{Ali}$ and Mohajir ${ }^{12}$ and Shetty et al. ${ }^{13}$ DPA deviated laterally in such cases and is very likely to be missed during manual examination pedal pulse. Vasudha and Ramesh ${ }^{6}$ mention similar lateral deviation in $6 \%$ of the cases (-Table 2 ).

Table 2 Comparative analysis of previous studies

\begin{tabular}{|c|c|c|c|c|c|c|c|}
\hline Author & $\begin{array}{l}\text { ATA } \\
\text { continues } \\
\text { as DPA }\end{array}$ & $\begin{array}{l}\mathrm{DPA}=\mathrm{PBPA} \\
\text { and ATA }\end{array}$ & $\begin{array}{l}\text { DPA branch } \\
\text { of PBPA }\end{array}$ & $\begin{array}{l}\text { ATA } \\
\text { Terminated }\end{array}$ & $\begin{array}{l}\mathrm{AA}-\mathrm{P} / \mathrm{A}, \\
\%\end{array}$ & $\begin{array}{l}\text { AA branch } \\
\text { of }\end{array}$ & $\begin{array}{l}\text { FDMA branch } \\
\text { of }\end{array}$ \\
\hline Swathi $(2010)^{2}$ & $74 \%$ & $4 \%$ & $16 \%$ & $8 \%$ & - & - & - \\
\hline Yamada et al. $(1993)^{3}$ & $86 \%$ & - & $6.70 \%$ & $9.10 \%$ & $\mathrm{~A}-33 \%$ & - & DPA-93\% \\
\hline $\begin{array}{l}\text { Vijayalakshmi et al. } \\
(2011)^{4}\end{array}$ & $92 \%$ & - & $8 \%$ & - & P-76\% & - & - \\
\hline $\begin{array}{l}\text { Rajeshwari et al. } \\
(2013)^{5}\end{array}$ & $54.76 \%$ & - & - & $9.52 \%$ & A- $16.6 \%$ & - & - \\
\hline $\begin{array}{l}\text { Vasudha and } \\
\text { Ramesh }(2012)^{6}\end{array}$ & $24.10 \%$ & $12.10 \%$ & - & - & - & - & - \\
\hline Present study & $86 \%$ & $6 \%$ & $2 \%$ & $6 \%$ & $\begin{array}{l}\text { P-90\%, } \\
\text { A-10\% }\end{array}$ & $\begin{array}{l}\text { DPA-86\%, } \\
\text { Loop-2\%, } \\
\text { PBPA- } 2 \%\end{array}$ & $\begin{array}{l}\text { DPA-96\%, } \\
\text { LTA-2\%, } \\
\text { Loop-2\% }\end{array}$ \\
\hline Dilandro et al. $(2001)^{7}$ & - & - & - & - & P- $16.7 \%$ & - & - \\
\hline El Saeed et al. $(2008)^{8}$ & - & - & - & - & - & $\begin{array}{l}\text { DPA-90\%, } \\
\text { LTA -10\% }\end{array}$ & - \\
\hline Lee and Dauber $(1997)^{9}$ & - & - & - & - & - & - & $\begin{array}{l}\text { DPA- } 90.6 \% \text {, } \\
\text { LTA-9.4\% }\end{array}$ \\
\hline $\begin{array}{l}\text { Gabrielli and } \\
\text { Olave }(2008)^{10}\end{array}$ & - & - & - & - & - & - & DPA- $86 \%$ \\
\hline
\end{tabular}

Abbreviations: AA, P/A, Arcuate Artery, Present /Absent; ATA, Anterior Tibial artery; DPA, dorsalis pedis artery; FDMA, First Dorsal Metatarsal Artery; Loop, Loop formed by ATA and PBPA; LTA, Lateral Tarsal Artery; PBPA, Perforating Branch of Peroneal Artery. 
ATA terminated as a medial and a lateral branch in $8 \%$ and DPA was the branch of lateral branch in $6 \%$ and medial branch in $2 \%{ }^{2}$ Vasudha and Ramesh ${ }^{6}$ mention termination of ATA by bifurcation in larger LTA and smaller DPA in 9.1\%. Termination of ATA by bifurcation in MTA and LTA in 9.52\% cases was noted by Rajeshwari et al. (2013). ${ }^{5}$ In our study ATA terminated in MTA and LTA in 6\% of the limbs, DPA was a branch of LTA $2 \%$ limbs and MTA in $4 \%$ limbs. Vijayalakshmi and Varsha $^{14}$ reports a case of double DP artery where ATA trifurcated in LTA and two DP arteries, which dipped in to first metatarsal space. Lateral of the two continued as plantar arch and medial one continued as FDMA. We have also encountered trifurcation of ATA in $2 \%$ of limbs in our study. However the branching pattern was different. The three branches at termination of ATA were lateral malleolar artery, lateral tarsal artery and dorsalis pedis artery. FDMA was a branch of DP, AA was absent and all other dorsal metatarsal arteries were branches of LTA (-Table 2 ).

Our study shows variation in branching pattern of DPA in $18 \%$ of limbs in form of absent AA in $10 \%$. FDMA was not a branch of DPA in 4\% and in 4\% DPA gave SDMA which normally is a branch of Arcuate artery (AA) (-Table 2).

AA was present in $76 \%,{ }^{4}$ AA was absent in $33 \%{ }^{3}$ where as AA was found only in $16.7 \%$ cadavers studied by Dilandro et al. ${ }^{7}$ AA was absent in $16.6 \%$ cases studied by Rajeshwari et al. ${ }^{5}$ and in $2.38 \%$ of these cases AA was replaced by loop formed by two LTA that gave branches to second and third and fourth dorsal metatarsal arteries. AA was branch of DPA in $90 \%$ and LTA in $10 \% .{ }^{8}$ In our study, AA was present in $90 \%$ and absent in $10 \%$. When present, AA was branch of DPA in $86 \%$, branch of loop formed by LTA and MTA in $2 \%$ and was a branch of PBPA in $2 \%$ of the limbs. Absence of AA ranges from $10 \%$ in our study, $16.6 \%$ by Rajeshwari et al., ${ }^{5} 33 \%^{3}$ to $83.5 \%$ by Dilandro et al. ${ }^{7}$ (- Table 2).

FDMA was branch of DP in 93\%, ${ }^{3}$ FDMA from DP in $90.6 \%$ and LTA in $9.4 \%$ as mentioned by Lee and Dauber, ${ }^{9}$ FDMA is branch of DP in $86 \%$ by Gabrielli and Olave. ${ }^{10}$ FDMA was branch of DPA in $96 \%$ in our study, when FDMA was not a branch of DPA, it was branch of LTA in $2 \%$ and of loop formed by ATA and PBPA in 2\%. 2nd to 4th DMA were not branchs of AA in $12 \%$ but they were branches of LTA and from loop formed by ATA and PBPA ( - Table 2 ).

Vasudha and Ramesh ${ }^{6}$ mention that only $15.2 \%$ cases showed normal branching pattern of DPA on dorsum of foot. From all the studies mentioned above it is evident that DPA has lot of variation in its branching pattern and size. DPA was absent in $4.2 \%,{ }^{15} 10 \%{ }^{16}$ and $10-12 \%$ as found by Vijayalakshmi et al. ${ }^{4}$ We have found that DPA was hypoplastic and thin and difficult to dissect when it was a branch either of MTA and LTA, it deviated laterally when it was continuation of PBPA and had variable in position when it was a branch of loop formed from ATA and PBPA. All these limbs also showed variable branching pattern of DPA, Variation in origin of DPA when associated with absence of AA, most common finding in such cases is FDMA is a branch of DPA and remaining dorsal metatarsal arteries rise from loop formed by ATA and PBPA or from LTA.

LTA was constantly found in all the 50 dissected limbs in present study. It travelled laterally and supplied extensor digitorum brevis musle and anastomosed with branches of lateral malleolar and lateral plantar arteries. Absence of LTA has not been mentioned in any of the studies mentioned above. On the contrary occurrence of three LTA are mentioned by Rajeshwari et al. (2013). ${ }^{5}$ They found three LTA in $2.38 \%$ and two LTA in $14.29 \%$. Presence of two LTA is also reported by Hamada et al. (1993) ${ }^{17}$ and also EL SAEED E M et al (2008). ${ }^{8}$ From all the studies available in literature it is evident that there exists a strong association related to variation in termination of ATA and branching pattern and origin of DPA. In such cases blood supply to arterial tree on dorsum of foot is supplemented by LTA. Taking this in to consideration authors feel that, in addition to palpation of DPA, should the pulsation of LTA be routinely performed at the proximomedial edge of first muscle belly of Extensor digitorum brevis either manually or by doppler probe on the lateral aspect of navicular ${ }^{18}$ needs to be discussed and evaluated. Authors are of the opinion that recording the pulsations of Lateral tarsal artery should be a part of routine clinical examination.

DP is a versatile vessel used for assessing pedal pulse as a part of assessment of PVD, for calculation of ABPI which is an important marker of cardiovascular disease. Change in value of ABPI is crucial for assessing the progress of PVD and in treatment of foot ulcers. DPA is used as a vascular pedicle for myocutaneous grafts of EDB muscle. ${ }^{19}$ In absence of proximal vessels, DP is used for arterial reconstruction to vascularize distal foot as an effort to salvage the ischemic lower extremity in diabetic patients thereby avoiding limb amputation. $^{20}$ DPA, LTA and plantar arteries are used for envdovascular interventions like percutaneous translumial angioplasty and stent placement in case of morbid patients where surgical bypass is contraindicated. Martin et al. ${ }^{21}$ quotes that DPA can be used for long term cannulation in ICU patients because long term cannulation of DP holds similar risks to that of radial artery and has advantage when immobilization of patients hand is undesirable or radial artery is inaccessible due to extensive trauma, burns, or damage from previous catheterizations. Direct vascularization of the artery feeding the ischemic angiosome is considered more beneficial because of better wound healing thus reducing the chances of amputation. ${ }^{22}$ Presence of Lateral calcaneal artery has been demonstrated by Chang et al. ${ }^{23}$ and Zeng-Yuan et al. ${ }^{24}$ They have also used this vessel as a recipient pedicle in foot reconstruction without sacrificing main circulation.

ATA and DP is easily accessible for palpation of pedal pulse however their variation and or absence as documented by various studies in literature warrants sole dependency on DP for its use in various clinical procedures with special reference to limb salvage surgery or Myocutaneous grafts. To have more clarity about the anatomy of arterial tree on dorsum of foot which would definitely help clinicians for direct revascularization of ischemic artery specific angiosome or during assessment of PVD, we plan to study the anatomy of lateral arterial chain and planter network of foot as an extension and addition to our study of angiosome formed by Anterior tibial artery and Dorsalis pedis artery. 


\section{Conclusion}

Anterior tibial artery is used to calculate ABPI (Ankle brachial pressure index). ABPI is a marker of cardiovascular disease and crucial parameter in treatment of peripheral vascular diseases and management of vascular ulcers of leg and foot. The branching of arterial tree on the dorsum of foot is helpful while planning Myocutaneous flaps for reconstruction surgeries of the foot during treatment of thromboanginitis obliterans of burgers disease, micro vascular neuropathy of diabetes mellitus, vehicular accidents and other soft tissue and bone defects. Knowledge of variation in Dorsalis pedis artery is necessary for revascularization of reconstitution distal foot vessels is main motive of diabetic foot surgery. Authors are of opinion that, pedal pulsation should be routinely assessed in Lateral tarsal artery in addition to Dorsalis pedis artery. Variations in DP artery must be taken in to account while assessing peripheral pulsations as variable branching pattern of dorsalis pedis artery leading to its variable position is responsible for diminished or weak Dorsalis pedis pulsation which is an important clinical indicator of peripheral vascular disease.

\section{References}

1 Attinger CE, Evans KK, Bulan E, Blume P, Cooper P. Angiosomes of the foot and ankle and clinical implications for limb salvage: reconstruction, incisions, and revascularization. Plast Reconstr Surg 2006;117 (7, Suppl)261S-293S. Doi: 10.1097/01.prs.0000222582.84385.54

2 Swathi A. A morphological study of extensor digitorum brevis muscle of the foot in human cadavers. Banglore: Rajiv Gandhi University of Healthsciences. Dissertation; 2010:95

3 Yamada T, Gloviczki P, Bower TC, Naessens JM, Carmichael SW. Variations of the arterial anatomy of the foot. Am J Surg 1993;166 (02):130-135, discussion 135

4 Vijayalakshmi S, Gunapriya R, Varsha S. Anatomical study of dorsalis pedis artery and its clinical correlations. J Clin Diagn Res 2011;5(02):287-290

5 Rajeshwari MS, Roshankumar BN. Vijayakumar. An anatomical study on dorsalis pedis artery. Inter J Anatom Research 2013;1(02):88-92

6 Vasudha K, Ramesh BR. A morphological study of dorsalis pedis artery and its clinical correlation. J Pharm Biolog Sciences 2012;2 (03):14-19
7 DiLandro AC, Lilja EC, Lepore FL, et al. The prevalence of the arcuate artery: a cadaveric study of 72 feet. J Am Podiatr Med Assoc 2001;91(06):300-305

8 El Saeed EM, El Monsif AA, El Sayed MA, Aly NM, Gezlan NA. Anatomical study of the Dorsalis pedis artery and its surgical importance in reconstructive surgeries. Allexandria Bulletin 2008;44(02):557-571

9 Lee JH, Dauber W. Anatomic study of the dorsalis pedis-first dorsal metatarsal artery. Ann Plast Surg 1997;38(01):50-55

10 Gabrielli C, Olave E. Origins of dorsal metatarsal arteries in Humans. Scand J Plast Reconstr Surg Hand Surg 2008;42(04): 174-181

11 Tuncel M, Maral T, Celik H, Tasçioglu B. A case of bilateral anomalous origin for dorsalis pedis arteries (anomalous dorsalis pedis arteries). Surg Radiol Anat 1994;16(03):319-323

12 Ali MW, Mohajir AM. Dorsalis pedis artery: variations and clinical significance. J Indian Med Assoc 1996;94(11):417-418

13 Shetty SD, Nayak S, Kumar N, Abhinitha P. Hypoplastic anterior tibial artery associated with continuation of fibular (peroneal) artery as dorsalis pedis artery: a case report. Int J Morphol 2013; 31(01):136-139

14 Vijayalakshmi S, Varsha S. Double dorsalis pedis artery: a rare case report. Intern J Anatom Sciences 2010;1:37-38

15 Reich RS. The pulses of the foot: their value in the diagnosis of peripheral circulatory disease. Ann Surg 1934;99(04):613-622

16 Legel K, Savard M, Blanco CJ, Jayanetti C. Dorsalis pedis aneurysm: a case report and review of the literature. J Foot Ankle 1998;1(11):1

17 Hamada N, Ikuta Y, Ikeda A. Arteriographic study of the arterial supply of the foot. Surg Radiol Anat 1993;15:187-192

18 Kunnamo I. Doppler stethoscopy in diagnostics: evidence based medicine guidelines. Chap. 5. Chichester: John Wiley \& Sons; 1993

19 del Piñal F, Herrero F. Extensor digitorum brevis free flap: anatomic study and further clinical applications. Plast Reconstr Surg 2000;105(04):1347-1356

20 Aulivola B, Pomposelli FB. Dorsalis pedis, tarsal and plantar artery bypass. J Cardiovasc Surg (Torino) 2004;45(03):203-212

21 Martin C, Saux P, Papazian L, Gouin F. Long-term arterial cannulation in ICU patients using the radial artery or dorsalis pedis artery. Chest 2001;119(03):901-906

22 Neville RF, Attinger CE, Bulan EJ, Ducic I, Thomassen M, Sidawy AN. Revascularization of a specific angiosome for limb salvage: does the target artery matter? Ann Vasc Surg 2009;23(03):367-373

23 Chang H, Kwon SS, Minn KW. Lateral calcaneal artery as a recipient pedicle for microsurgical foot reconstruction. J Plast Reconstr Aesthet Surg 2010;63(11):1860-1864

24 Zeng-Yuan S, Wei-Gang Y, Hai-Jiao M, Fa-Ke W, Yong-Sheng F. Applied anatomy of the island flap pedicled with lateral pedis dorsal artery chain. Chinese J Clin Anatom 2012;30(06):609-611 